\title{
Absorption and Laser Induced Fluorescence Spectroscopy of Neutral Polycyclic Aromatic Hydrocarbons in Argon matrices
}

\author{
Salma Bejaoui ${ }^{1,2}$, Farid Salama ${ }^{1}$ and Ella Sciamma-O'Brien ${ }^{1,3}$ \\ ${ }^{1}$ NASA Ames Research Center, Mail Stop 245-6, Moffett Field, California 94035-1000 \\ ${ }^{2}$ NPP, Oak Ridge Associated Universities ${ }^{3}$ BAER Institute, Petaluma, CA \\ email: salma.bejaoui@nasa.gov
}

Keywords. PAH, fluorescence, UV-VIS, ERE, comet.

Polycyclic aromatic hydrocarbons (PAHs) are considered as plausible carriers for the extended red emission (ERE), a photoluminescent process associated with a wide variety of interstellar environments, as well as for broad emission band features seen in cometary spectra. We report the absorption spectra of phenanthrene, anthracene, fluoranthene, pentacene, pyrene, chrysene and triphenylene isolated at $10 \mathrm{~K}$ in solid argon matrices together with laser induced fluorescence (LIF) spectra at $355 \mathrm{~nm}$ of matrix-isolated anthracene and fluoranthene. LIF spectra are compared with the UV/blue fluorescence spectra of the Red Rectangle Nebula (RR). The LIF spectra measured in solid Ar matrices have been shifted to the predicted position of the PAH band emission in the gas phase for comparison with the astronomical observations (Fig. 1). These preliminary results indicate that small neutral PAHs can well account for the blue fluorescence observed in the RR as it has been previously proposed (Vijh, et al. (2004)). LIF spectra of anthracene measured in Ar matrices are also compared to the emission spectra of $1 \mathrm{P} / \mathrm{Halley}$ 's inner coma(Fig. 2). LIF spectra are shifted to fit the fluorescence of anthracene measured in the gas phase. Four main bands in the spectrum of anthracene peak at 363, 367.5, 373 and $382.5 \mathrm{~nm}$ and coincide with the spectral features observed at 363, 367.5, 373 and $382.5 \mathrm{~nm}$ in the emission spectra of $1 \mathrm{P} /$ Halley's inner coma as reported by Clairemidi, et al. (2008). The purpose of this study is to complement the extensive PAH absorption spectroscopy database that has been developed over the past years (Salama (2008)) with emission spectroscopy data.

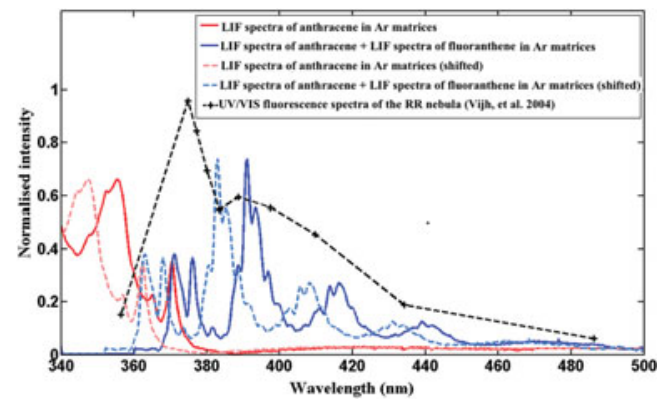

Figure 1. LIF spectra of anthracene and fluoranthene compared to the RR Nebula emission. The spectra have been shifted to account for the matrix-to-gas phase shift.

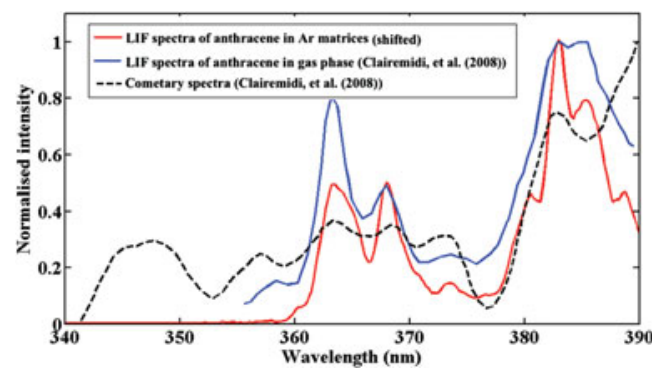

Figure 2. LIF spectra of anthracene compared to the $1 \mathrm{P} /$ Halley's inner coma emission. The spectra have been shifted to account for the matrix-to-gas phase shift.

\section{References}

Vijh, U. P., Witt, A. N., \& Gordon, K. D. 2004, ApJ , 606:L65-L68

Clairemidi, J., Moreels, G., Mousis, O., \& Bréchignac, P. 2008, A\& A, 492, 245

Salama,F., 2008, In Organic Matter in Space, IAU Symposium 251, KwokESSandford Eds. Cambridge University Press, Vol. 4, S251, p. 357 and references therein. 